\title{
Identification of nondiabetic heart failure-associated genes by bioinformatics approaches in patients with dilated ischemic cardiomyopathy
}

\author{
ANZHONG YU ${ }^{1}$, JINGYAO ZHANG ${ }^{2}$, HAIYAN LIU ${ }^{3}$, BING LIU ${ }^{1}$ and LINGDONG MENG ${ }^{1}$ \\ ${ }^{1}$ Department of Cardiology, Jinan No. 4 People's Hospital, Jinan, Shandong 250031; \\ ${ }^{2}$ Department of Blood Purification, Jinan Infectious Disease Hospital, Jinan, Shandong 250021; \\ ${ }^{3}$ Department of Internal Medicine, Jinan Minzu Hospital, Jinan, Shandong 250014, P.R. China
}

Received January 26, 2015; Accepted March 3, 2016

DOI: $10.3892 /$ etm.2016.3252

\begin{abstract}
Heart failure (HF) is a common pathological condition affecting $4 \%$ of the worldwide population. However, approaches for predicting or treating nondiabetic $\mathrm{HF}$ (ND-HF) progression are insufficient. In the current study, the gene expression profile GSE26887 was analyzed, which contained samples from 5 healthy controls, 7 diabetes mellitus-HF patients and $12 \mathrm{ND}-\mathrm{HF}$ patients with dilated ischemic cardiomyopathy. The dataset of 5 healthy controls and 12 ND-HF patients was normalized with robust multichip average analysis and the differentially expressed genes (DEGs) were screened by unequal variance t-test and multiple-testing correction. In addition, the protein-protein interaction (PPI) network of the upregulated and downregulated genes was constructed using the Search Tool for the Retrieval of Interacting Genes/Proteins database and the Cytoscape software platform. Subsequently, gene ontology (GO) and Kyoto Encyclopedia of Genes and Genomes (KEGG) pathway enrichment analyses were performed. A total of 122 upregulated and 133 downregulated genes were detected. The most significantly up- and downregulated genes were EIFIAY and SERPINE1, respectively. In addition, 38 and 77 nodes were obtained in the up- and downregulated PPI network. DEGs that owned the highest connectivity degree were USP9Y and $U T Y$ in the upregulated network, and CD44 in the downregulated networks, respectively. NPPA and SERPINE1 were also found to be hub genes in the PPI network. Several GO terms and pathways that were enriched by DEGs were identified, and the most significantly enriched KEGG pathways were drug metabolism and extracellular matrix-receptor interaction. In
\end{abstract}

Correspondence to: $\mathrm{Dr}$ Anzhong Yu, Department of Cardiology, Jinan No. 4 People's Hospital, 50 Shifan Road, Jinan, Shandong 250031, P.R. China

E-mail: anzhongyy@163.com

Key words: heart failure, differentially expressed genes, protein-protein interaction network, gene ontology, pathway conclusion, the two DEGs, NPPA and SERPINE1, may be important in the pathogenesis of HF and may be used for the diagnosis and treatment of HF.

\section{Introduction}

Heart failure (HF), also known as chronic HF, is one of the major causes of mortality affecting approximately $4 \%$ of the world's population, while the prevalence of this condition is currently increasing $(1,2)$. In addition to the widely known risk factor of glucose abnormalities (observed in $~ 43 \%$ of $\mathrm{HF}$ patients) (3), HF can also result from certain other factors, which is classified as nondiabetic HF (ND-HF) and is based on a complicated pathological mechanism (4,5). Since HF greatly affects human health and has an unclear pathogenesis, numerous studies have investigated this condition.

Previous studies have proposed certain markers associated with HF. For instance, hyperuricemia and elevated levels of circulating markers of inflammation are common in HF $(6,7)$, and thus the inflammatory biomarker YKL-40 was investigated and found to be significantly associated with all-cause mortality in patients with HF (8). In addition, as a marker of cardiomyocyte injury, cardiac troponin $\mathrm{T}$ is a predictor of adverse outcomes for patients with chronic HF (9). Troughton et al (10) observed that patients with impaired systolic function or symptomatic HF could be treated under N-terminal brain natriuretic peptide (N-BNP) guidance to partly reduce the total number of cardiovascular events. Despite vast efforts to predict and prevent HF in order to decrease the morbidity and mortality associated with this condition, there is no clear division between ND-HF and diabetic HF. Furthermore, simple and reliable measurements to diagnose this disease earlier and to effectively predict the prognosis remain insufficient.

In the current study, the gene expression profiles generated from healthy controls and ND-HF patients were analyzed. Biopsy tissues were collected during the surgical ventricular restoration in patients with dilated hypokinetic ischemic cardiomyopathy. Differentially expressed genes (DEGs) were screened and their possible roles in the pathogenesis of HF were explored using multiple bioinformatics methods. The 
main aim of the present study was to identify better markers for the diagnosis and treatment of ND-HF.

\section{Materials and methods}

Microarray dataset. The microarray dataset under the accession number GSE26887 (11) were obtained from the Gene Expression Omnibus (12) database (http://www.ncbi. nlm.nih.gov/geo/) of the National Center for Biotechnology Information (Bethesda, MD, USA). The gene expression profile was generated based on the platform GPL6244 (Affymetrix Human Gene 1.0 ST Array; Affymetrix, Inc., Santa Clara, CA, USA). This dataset was derived from RNA samples extracted from 12 ND-HF patients (12 males) and 5 healthy controls (2 males, 3 females). Myocardial biopsy samples were collected from the vital, non-infarcted zone of left ventricular of patients with dilated ischemic hypokinetic cardiomyopathy during surgical ventricular restoration procedures (11). In addition, left ventricle cardiac biopsy samples were collected by Greco et al (11) from the vital, non-infarcted zone of control patients who had succumbed to mortality (as a result of non-cardiac associated causes), within $<24 \mathrm{~h}$.

Data preparation and DEGs screening. Robust multichip average (RMA) (13), which contained three steps including background adjustment, quantile normalization and summarization, was used as a probe set algorithm. The original dataset and the annotation file of the platform were preprocessed using the RMA method of the BioConductor Oligo package (version 2.12; www.bioconductor.org). Probe set IDs were transformed into gene symbols, and the gene expression matrix was constructed.

Statistically significant differences in the expression levels of the various genes were first calculated by the unequal variance t-test, and was then adjusted for multiple testing using the Benjamini and Hochberg procedure (14). After comparing the expression of these genes in the control and HF tissues, the adjusted P-value was obtained, and DEGs with an adjusted P-value of $<0.05$ and a $\mid \log _{2}$ fold change (FC) $\mid$ of $>1$ were screened and were considered as potential HF-associated genes.

Protein-protein interaction (PPI) network. The Search Tool for the Retrieval of Interacting Genes/Proteins (STRING) (15) is a widely used database that includes the known and predicted protein interactions. PPI network analysis of the upregulated and downregulated DEGs was performed utilizing the STRING online tool. A confidence (combined) score of $>0.4$ was selected as the threshold of PPIs.

The PPI network was constructed using the Cytoscape software platform (16) based on the PPI associations identified. Since the vast majority of biological networks are subject to the scale-free (without scale) properties of the network, the connectivity degree was used for the analysis of important nodes (hub proteins) in the PPI network $(17,18)$.

Gene ontology (GO) and pathway analysis. The Kyoto Encyclopedia of Genes and Genomes (KEGG) (19) is an authoritative database containing a variety of biochemical pathways. In addition, the Database for Annotation, Visualization and Integration Discovery (DAVID) (20) is a gene functional classification tool that organizes and condenses abundant
Table I. Top 10 upregulated and downregulated genes.

\begin{tabular}{lll}
\hline Gene & $\log _{2} \mathrm{FC}$ & Adjusted P-value \\
\hline Upregulated & & \\
EIF1AY & 3.412 & 0.00281 \\
NPPA & 3.115 & 0.00038 \\
DSC1 & 2.445 & 0.00031 \\
NEB & 2.418 & 0.00545 \\
MYL4 & 2.346 & 0.00204 \\
UTY & 2.254 & 0.00414 \\
FRZB & 2.171 & 0.00165 \\
USP9Y & 2.095 & 0.00550 \\
SLN & 2.092 & 0.00712 \\
RBMY1A1 & 1.993 & 0.00038 \\
Downregulated & & \\
SERPINE1 & -3.182 & 0.00301 \\
SERPINA3 & -2.904 & 0.00037 \\
TNC & -2.223 & 0.01926 \\
SPP1 & -2.129 & 0.01003 \\
CYP1B1 & -2.027 & 0.00858 \\
SIO0A8 & -1.955 & 0.00130 \\
ANKRD2 & -1.903 & 0.00079 \\
GFPT2 & -1.824 & 0.00020 \\
MYC & -1.821 & 0.00117 \\
CD163 & -1.805 & 0.00022 \\
\hline
\end{tabular}

$\log _{2} \mathrm{FC}, \log _{2}$-transformed fold change of gene expression.

heterogeneous annotation content. Functional enrichment analysis was conducted in order to recognize the DEG enriched biochemical pathways using KEGG database and GO-associated biological functions. Furthermore, DAVID online tools were applied for the GO and KEGG pathway enrichment analyses with a P-value set to $<0.05$.

\section{Results}

DEG screening. A significant gender difference of the sample source existed between the control and ND-HF subjects; thus, gender-correlated results were carefully considered or abandoned. A total of 255 DEGs were obtained in the ND-HF patients, including 122 upregulated and 133 downregulated genes. As shown in Table I, the EIF1AY, NPPA and DSC1 were the three most upregulated genes. Similarly, the three most downregulated genes were SERPINE1, SERPINA3 and $T N C$, and their respective $\log _{2}$ FC values were $-3.182,-2.904$ and -2.223 (Table I).

PPI network. In total, 38 nodes and 53 node-pairs were identified in the PPI network of the upregulated DEGs. Furthermore, 77 nodes and 149 node-pairs were obtained in the PPI network of downregulated DEGs. Subsequent to filtering out the low-degree nodes and nodes without connections, the up- and downregulated PPI networks were constructed, as shown in Figs. 1 and 2, respectively.

Tables II and III exhibited the connectivity degree of the top $30 \%$ nodes in the PPI network of upregulated and 


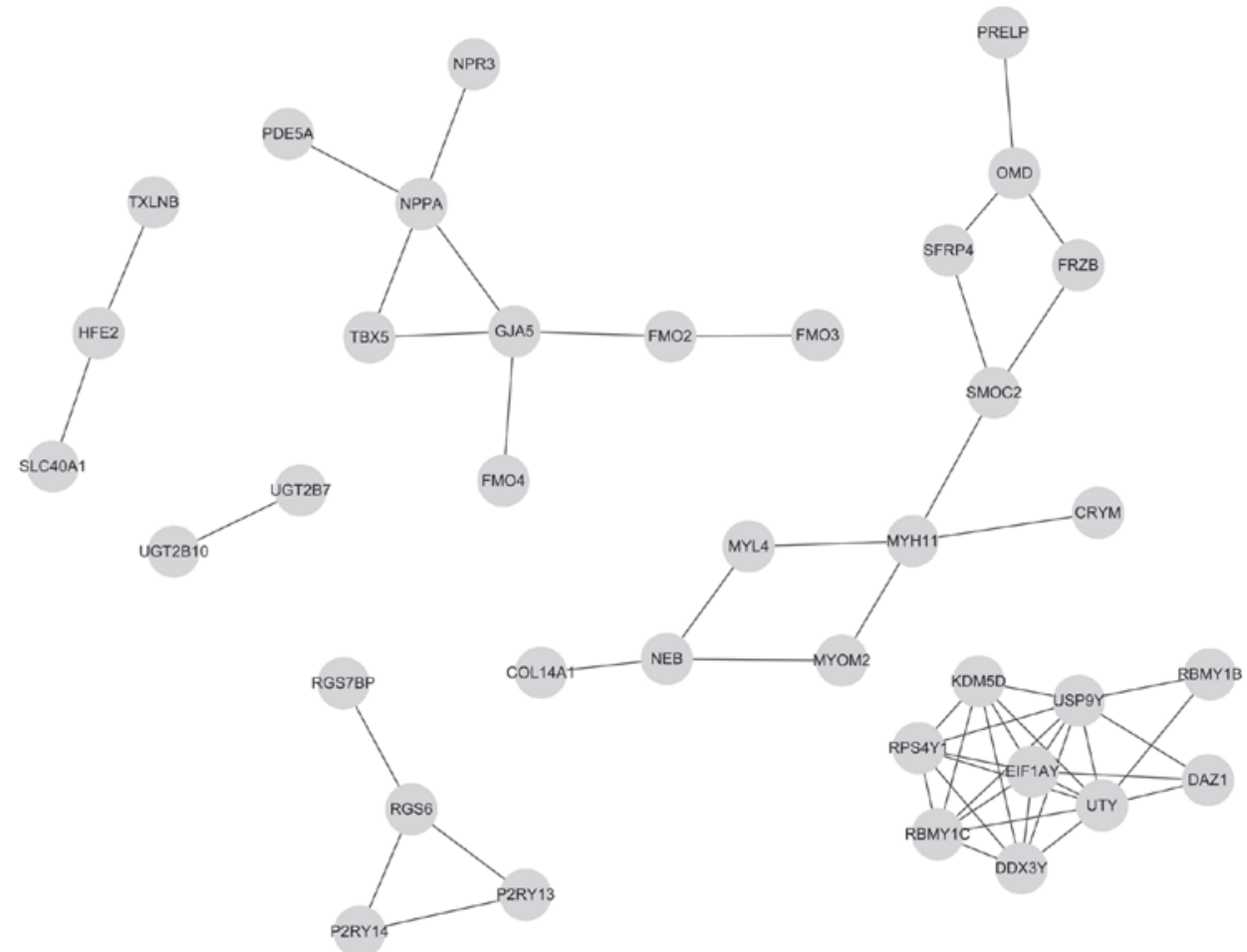

Figure 1. Upregulated differentially expressed genes in the protein-protein interaction network.

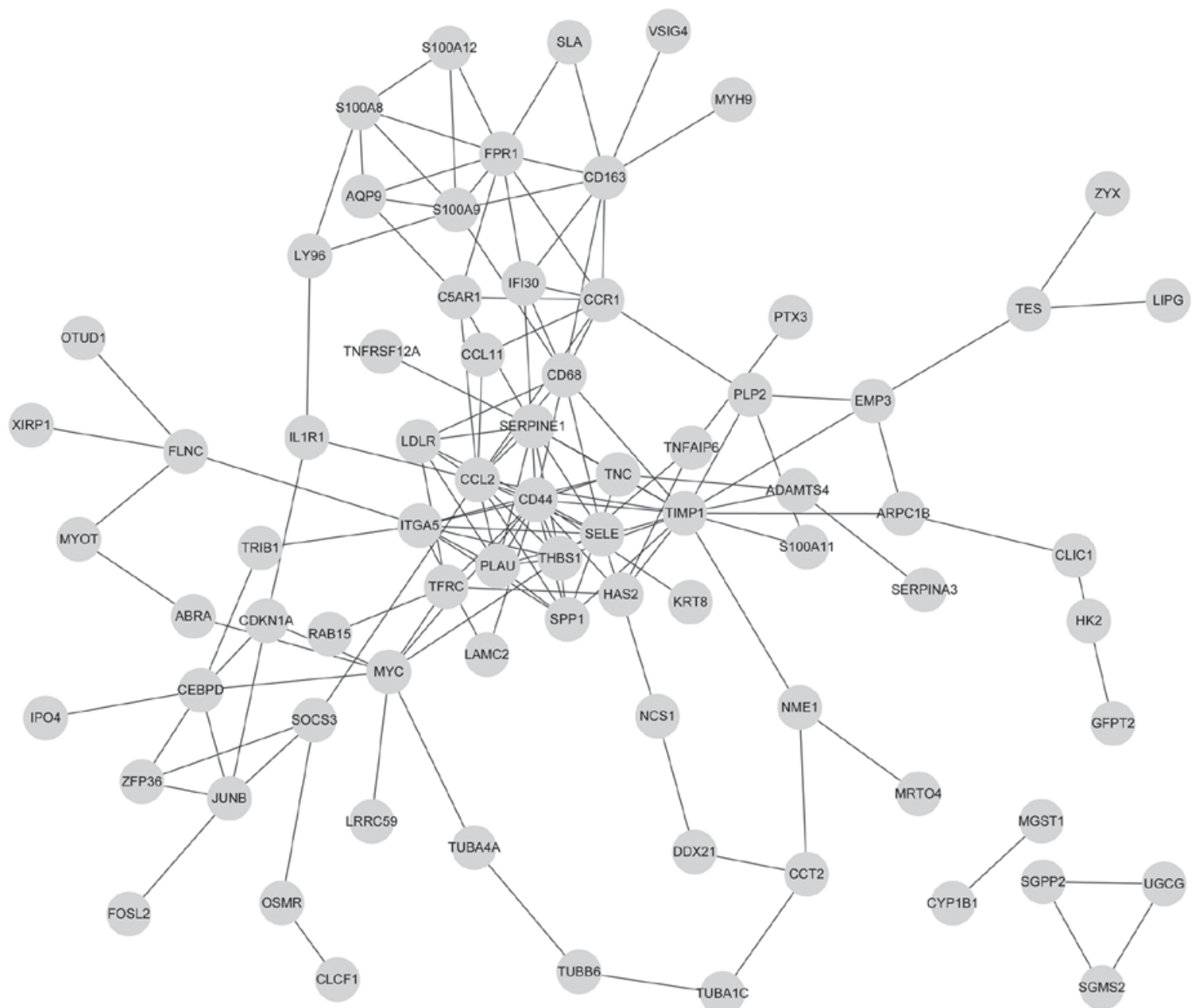

Figure 2. Downregulated differentially expressed genes in the protein-protein interaction network. 
Table II. Top $30 \%$ of the node connections in the upregulated protein-protein interaction network.

\begin{tabular}{lc}
\hline Gene & Degree \\
\hline USP9Y & 8 \\
UTY & 8 \\
EIF1AY & 7 \\
DDX3Y & 6 \\
KDM5D & 6 \\
RBMY1C & 6 \\
RPS4Y1 & 6 \\
GJA5 & 6 \\
NPH11 $P A$ & 4 \\
\hline
\end{tabular}

Degree refers to the number of gene connections within the network.

Table III. Top $30 \%$ of the node connections in the downregulated protein-protein interaction network.

\begin{tabular}{|c|c|}
\hline Gene & Degree \\
\hline$C D 44$ & 16 \\
\hline TIMP1 & 15 \\
\hline CCL2 & 14 \\
\hline THBS1 & 9 \\
\hline SERPINE1 & 9 \\
\hline FPR1 & 9 \\
\hline CD68 & 9 \\
\hline ITGA5 & 9 \\
\hline CCR1 & 8 \\
\hline$C D 163$ & 8 \\
\hline$M Y C$ & 8 \\
\hline$P L A U$ & 8 \\
\hline SPP1 & 7 \\
\hline S100A9 & 7 \\
\hline$C E B P D$ & 6 \\
\hline SELE & 6 \\
\hline$T N C$ & 6 \\
\hline$L D L R$ & 6 \\
\hline C5AR1 & 5 \\
\hline IFI30 & 5 \\
\hline TFRC & 5 \\
\hline S100A8 & 5 \\
\hline$J U N B$ & 5 \\
\hline
\end{tabular}

Degree refers to the number of gene connections within the network.

downregulated DEGs, respectively. According to the calculation results, the connectivity degree of each node was $>4$ in the upregulated and downregulated networks. The connectivity degree of $N P P A$ was 4 , without any connections with the USP9Y, UTY and EIFIAY genes. In the downregulated network, the top five nodes with a high connectivity degree were CD44, TIMP1, CCL2, THBS1 and SERPINE1.
GO and KEGG pathway analyses of DEGs. GO analysis revealed that the significantly-enriched GO terms of upregulated DEGs included muscle contraction, muscle system process, circulatory system process (involving NPPA), blood circulation, muscular organ development, male gamete generation, spermatogenesis, cGMP metabolic process and skeletal system development. In addition, the significantly enriched GO terms of downregulated DEGs were mainly associated with the stimulus response, response to bacterium and response to nutrient. NPPA was also involved in the GO term of regulation of cell growth (Table IV).

KEGG pathway analysis revealed that the significantly enriched pathways of upregulated DEGs were drug metabolism, ascorbate and aldarate metabolism, and pentose and glucuronate interconversions (Table V). By contrast, the significantly enriched pathways of the downregulated DEGs were extracellular matrix (ECM)-receptor interactions (involving the genes THBS1, CD44 and TNC), pathogenic Escherichia coli infection, focal adhesion (involving TNC and THBS1), cytokine-cytokine receptor interaction (involving CCL11 and CCL2), hematopoietic cell lineage (involving CD44), sphingolipid metabolism, and bladder cancer (involving THBS1; Table V).

\section{Discussion}

HF with fairly high morbidity and mortality (21), is increasing in prevalence with the aging of the worldwide population (22). In order to improve the understanding on the underlying mechanisms and identify molecular markers of $\mathrm{HF}$, particularly in dilated ischemic cardiomyopathy-associated HF, the present study screened the DEGs between control and ND-HF patients. In addition, these DEGs were used for PPI network construction, while GO and KEGG pathway analyses were also performed. A total of 122 upregulated and 133 downregulated genes were detected. The most significantly upregulated and downregulated genes were NPPA and SERPINE1, respectively. Furthermore, $N P P A$ and SERPINE1 were not only differentially expressed in ND-HF patients, but were also found to be hub nodes in the PPI network. Certain GO terms and KEGG pathways enriched by DEGs were obtained. Therefore, these hub genes and functional terms may be closely associated with ND-HF.

The protein encoded by the upregulated NPPA gene is the atrial natriuretic peptide (ANP), which is a member of the natriuretic peptide family that is involved in the control of the extracellular fluid volume and electrolyte homeostasis $(23,24)$. The GO term of circulatory system process, in which NPPA is involved, is vital for homeostasis. In addition, mutations in NPPA gene are linked to atrial fibrillation (25). In 1998, Maeda etal(26) stated that brain natriuretic peptide(BNP) levels were correlated with the left ventricular end-diastolic pressure. However, a more recent study by Seronde et al (27) found that the mid-regional sequence of pro-ANP (MR-proANP) has a more long term prognostic value when compared with BNP in patients with acute HF. Furthermore, Potocki et al (28) suggested that MR-proANP appears to provide incremental information superior to BNP in certain subgroups of patients. Notably, GO terms and KEGG pathways enriched by NPPA or other genes are essential in cardiac failure. Therefore, due to these advantages of ANP when compared with BNP, NPPA 
Table IV. Gene ontology term enrichment analyses of the differentially expressed genes.

\begin{tabular}{|c|c|c|c|}
\hline Category & GO-BP Term & Count & P-value \\
\hline \multicolumn{4}{|l|}{ Upregulated } \\
\hline GO:0006936 & Muscle contraction & 4 & 0.004604 \\
\hline GO:0003012 & Muscle system process & 4 & 0.005971 \\
\hline GO:0003013 & Circulatory system process & 4 & 0.007904 \\
\hline GO:0008015 & Blood circulation & 4 & 0.007904 \\
\hline GO:0007517 & Muscle organ development & 4 & 0.011139 \\
\hline GO:0048232 & Male gamete generation & 4 & 0.030149 \\
\hline GO:0007283 & Spermatogenesis & 4 & 0.030149 \\
\hline GO:0046068 & cGMP metabolic process & 2 & 0.030618 \\
\hline GO:0001501 & Skeletal system development & 4 & 0.032968 \\
\hline \multicolumn{4}{|l|}{ Downregulated } \\
\hline GO:0009611 & Response to wounding & 21 & $5.31 \times 10^{-13}$ \\
\hline GO:0006954 & Inflammatory response & 17 & $3.16 \times 10^{-12}$ \\
\hline GO:0006952 & Defense response & 20 & $7.41 \times 10^{-11}$ \\
\hline GO:0032496 & Response to lipopolysaccharide & 6 & $3.84 \times 10^{-5}$ \\
\hline GO:0002237 & Response to molecule of bacterial origin & 6 & $6.54 \times 10^{-5}$ \\
\hline GO:0009617 & Response to bacterium & 7 & $3.78 \times 10^{-4}$ \\
\hline GO:0009991 & Response to extracellular stimulus & 8 & $1.04 \times 10^{-4}$ \\
\hline GO:0031667 & Response to nutrient levels & 7 & $4.22 \times 10^{-4}$ \\
\hline GO:0007584 & Response to nutrients & 6 & $6.38 \times 10^{-4}$ \\
\hline GO:0033273 & Response to vitamins & 4 & 0.004253 \\
\hline
\end{tabular}

$\mathrm{GO}$, gene ontology; BP, biological process.

Table V. Kyoto Encyclopedia of Genes and Genomes pathway analysis of the differentially expressed genes.

\begin{tabular}{|c|c|c|c|c|}
\hline Pathway term & Pathway description & Count & P-value & Associated genes \\
\hline $\begin{array}{l}\text { Upregulated } \\
\text { hsa00982 }\end{array}$ & Drug metabolism & 5 & $6.20 \times 10^{-6}$ & $\begin{array}{l}\text { FMO4, FMO2, FMO3, } \\
\text { UGT2B10, UGT2B7 }\end{array}$ \\
\hline hsa00053 & Ascorbate and aldarate metabolism & 2 & 0.036201 & $U G T 2 B 10, U G T 2 B 7$ \\
\hline hsa00040 & Pentose and glucuronate interconversions & 2 & 0.038293 & $U G T 2 B 10, U G T 2 B 7$ \\
\hline \multicolumn{5}{|l|}{ Downregulated } \\
\hline hsa04512 & Extracellular matrix-receptor interaction & 6 & $5.13 \times 10^{-4}$ & $\begin{array}{l}\text { CD44, ITGA5, TNC, } \\
\text { LAMC } 2, T H B S 1, \text { SPP1, } \\
\text { ARPC } 1 B, L Y 96,\end{array}$ \\
\hline hsa05130 & Pathogenic Escherichia coli infection & 5 & 0.001056 & $\begin{array}{l}\text { TUBB6, TUBA4A, } \\
\text { TUBA1C }\end{array}$ \\
\hline hsa04510 & Focal adhesion & 7 & 0.005006 & $\begin{array}{l}\text { ITGA5, TNC, LAMC2, } \\
\text { ZYX, FLNC, THBS1, SPP1 }\end{array}$ \\
\hline hsa04060 & Cytokine-cytokine receptor interaction & 7 & 0.017379 & $\begin{array}{l}\text { CCL11, IL1R1, } \\
\text { CCL2, TNFRSF } 12 A, \\
\text { OSMR, CLCF1, CCR } 1\end{array}$ \\
\hline hsa04640 & Hematopoietic cell lineage & 4 & 0.031377 & $\begin{array}{l}\text { ILIR1, TFRC, CD44, } \\
\text { ITGA5 }\end{array}$ \\
\hline hsa00600 & Sphingolipid metabolism & 3 & 0.038951 & $S G M S 2, S G P P 2, U G C G$ \\
\hline hsa05219 & Bladder cancer & 3 & 0.044581 & CDKN1A, THBS1, MYC \\
\hline
\end{tabular}

may also be an essential gene associated with ND-HF and may be used as a potential therapeutic target in ND-HF.

In addition to the poor contractility and low cardiac output, patients with HF also present with abnormal manifestations of platelets and endothelial dysfunction (29), while HF patients in sinus rhythm still present a higher thromboembolic risk (30). SERPINE1, also known as plasminogen activator inhibitor 1 (PAI-1) precursor, which was downregulated and pertained to 
the serine proteinase inhibitor superfamily, has a core effect in the regulation of fibrinolysis, coagulation, inflammation and neuromuscular patterning (31). Askari et al (32) hypothesized that genetic disruption of PAI-1 is essential in order to suppress ventricular remodeling in null mice with myocardial infarction; furthermore, PAI-1 is essential in microvascular integrity and cardiac homeostasis (33). Based on the results of the present and previous studies $(31,33)$, the plasma level of SERPINE1 is associated with thrombophilia and an increased risk of coronary artery disease (34). Therefore, SERPINE1 may be a useful marker for the diagnosis and treatment of ND-HF.

$\mathrm{HF}$ is accompanied by degradation of the collagen network of the ECM (35), and may subsequently cause heart dysfunction (36). Zheng et al (37) reported an overall decrease in the ECM-associated genes which are indispensable to the overall ECM structure and collagen assembly. Therefore, it is no surprise that $C D 44$, which is involved in the ECM-receptor interaction, was found to be downregulated in the current study. Chatila et al (38) also found that certain compositions of the infarct border zone may slow down left ventricular remodeling by suppressing inflammation. In the current study, GO terms enriched by downregulated genes were mainly associated with the stimulus and immune response. Considering these findings, the connections between ECM and inflammation participating in $\mathrm{HF}$ require further investigation, particularly in ND-HF.

In conclusion, based on the bioinformatics methods used in the current study, a number of DEGs were highlighted, particularly NPPA and SERPINE1, although the results were interfered by certain Y-linked genes to some extent. These two genes may be potential therapeutic targets and molecular markers contributing to improved prevention and treatment of cardiogenic disease. Additionally, the complicated correlation between ECM-protein expression and inflammation was further investigated. However, further comparison of these genes and those obtained from diabetic HF patients with dilated ischemic cardiomyopathy and controls is required to verify these results. Furthermore, gender-matched studies are needed, with a sufficiently large sample size. Future research should focus on these areas and verify these DEGs based on serum sample analysis.

\section{References}

1. Van Diepen S, Hellkamp AS, Patel MR, Becker RC, Breithardt G, Hacke W, Halperin JL, Hankey GJ, Nessel CC, Singer DE, et al Efficacy and safety of rivaroxaban in patients with heart failure and nonvalvular atrial fibrillation: Insights from ROCKET AF. Circ Heart Fail 6: 740-747, 2013.

2. Carr HJ, McDermott A, Tadbiri H, Uebbing A-M and Londrigan M: The effectiveness of computer-based learning in hospitalized adults with heart failure on knowledge, re-admission, self-care, quality of life and patient satisfaction: A systematic review protocol. The JBI Database of Systematic Reviews and Implementation Reports 11: 129-145, 2013.

3. Suskin N, McKelvie RS, Burns RJ, Latini R, Pericak D, Probstfield J, Rouleau JL, Sigouin C, Solymoss CB, Tsuyuki R, et al: Glucose and insulin abnormalities relate to functional capacity in patients with congestive heart failure. Eur Heart J 21: 1368-1375, 2000.

4. Diercks G, Van Boven A, Hillege H, Janssen W, Kors J, De Jong P, Grobbee D, Crijns H and Van Gilst W: Microalbuminuria is independently associated with ischaemic electrocardiographic abnormalities in a large non-diabetic population. The PREVEND (Prevention of REnal and Vascular ENdstage Disease) study. Eur Heart J 21: 1922-1927, 2000.
5. Klamann A, Sarfert P, Launhardt V, Schulte G, Schmiegel WH and Nauck MA: Myocardial infarction in diabetic vs non-diabetic subjects. Survival and infarct size following therapy with sulfonylureas (glibenclamide). Eur Heart J 21: 220-229, 2000.

6. Anker SD, Egerer KR, Volk H-D, Kox WJ, Poole-Wilson PA and Coats AJ: Elevated soluble CD14 receptors and altered cytokines in chronic heart failure. Am J Cardiol 79: 1426-1430, 1997.

7. Krishnan E: Hyperuricemia and incident heart failure. Circ Heart Fail 2: 556-562, 2009.

8. Harutyunyan M, Christiansen M, Johansen JS, Køber L, Torp-Petersen $\mathrm{C}$ and Kastrup J: The inflammatory biomarker YKL-40 as a new prognostic marker for all-cause mortality in patients with heart failure. Immunobiology 217: 652-656, 2012.

9. Kusumoto A, Miyata M, Kubozono T, Ikeda Y, Shinsato T, Kuwahata S, Fujita S, Takasaki K, Yuasa T and Hamasaki S: Highly sensitive cardiac troponin T in heart failure: Comparison with echocardiographic parameters and natriuretic peptides. J Cardiol 59: 202-208, 2012.

10. Troughton RW, Frampton CM, Yandle TG, Espiner EA, Nicholls MG and Richards AM: Treatment of heart failure guided by plasma aminoterminal brain natriuretic peptide (N-BNP) concentrations. The Lancet 355: 1126-1130, 2000.

11. Greco S, Fasanaro P, Castelvecchio S, D'Alessandra Y, Arcelli D, Di Donato M, Malavazos A, Capogrossi MC, Menicanti L and Martelli F: MicroRNA dysregulation in diabetic ischemic heart failure patients. Diabetes 61: 1633-1641, 2012.

12. Kapoun AM, Liang F, O'Young G, Damm DL, Quon D, White RT, Munson K, Lam A, Schreiner GF and Protter AA: B-type natriuretic peptide exerts broad functional opposition to transforming growth factor-beta in primary human cardiac fibroblasts: Fibrosis, myofibroblast conversion, proliferation and inflammation. Circ Res 94: 453-461, 2004.

13. IrizarryRA,Hobbs B,CollinF,Beazer-Barclay YD,AntonellisKJ, Scherf U and Speed TP: Exploration, normalization and summaries of high density oligonucleotide array probe level data. Biostatistics 4: 249-264, 2003.

14. Benjamini Y and Hochberg Y: Controlling the false discovery rate: A practical and powerful approach to multiple testing. Journal of the Royal Statistical Society. Series B (Methodological) 289-300, 1995.

15. Von Mering C, Huynen M, Jaeggi D, Schmidt S, Bork P and Snel B: STRING: A database of predicted functional associations between proteins. Nucleic Acids Res 31: 258-261, 2003.

16. Smoot ME, Ono K, Ruscheinski J, Wang PL and Ideker T: Cytoscape 2.8: New features for data integration and network visualization. Bioinformatics 27: 431-432, 2011.

17. He $X$ and Zhang J: Why do hubs tend to be essential in protein networks? PLoS Genet 2: e88, 2006.

18. Bader GD and Hogue CW: An automated method for finding molecular complexes in large protein interaction networks. BMC Bioinformatics 4: 2, 2003

19. Kanehisa M and Goto S: KEGG: Kyoto encyclopedia of genes and genomes. Nucleic Acids Res 28: 27-30, 2000.

20. Alvord WG, Roayaei J, Stephens R, Baseler MW, Lane HC, Lempicki RA, Huang DW, Sherman BT, Tan Q and Collins JR: The DAVID Gene Functional Classification Tool: A novel biological module-centric algorithm to functionally analyze large gene lists. Genome Biol 8: R183, 2007.

21. Bottomley PA, Panjrath GS, Lai S, Hirsch GA, Wu K, Najjar SS Steinberg A, Gerstenblith G and Weiss RG: Metabolic rates of ATP transfer through creatine kinase (CK Flux) predict clinical heart failure events and death. Sci Transl Med 5: 215re3, 2013.

22. Burchell AE, Sobotka PA, Hart EC, Nightingale AK and Dunlap ME: Chemohypersensitivity and autonomic modulation of venous capacitance in the pathophysiology of acute decompensated heart failure. Curr Heart Fail Rep 10: 139-146, 2013.

23. Tulassay T, Seri I and Rascher W: Atrial natriuretic peptide and extracellular volume contraction after birth. Acta Paediatr Scand 76: 444-446, 1987.

24. Samson WK: Atrial natriuretic factor inhibits dehydration and hemorrhage-induced vasopressin release. Neuroendocrinology 40: 277-279, 1985.

25. Ren X, Xu C, Zhan C, Yang Y, Shi L, Wang F, Wang C, Xia Y, Yang B, Wu G, et al: Identification of NPPA variants associated with atrial fibrillation in a Chinese GeneID population. Clin Chim Acta 411: 481-485, 2010.

26. Maeda K, Tsutamoto T, Wada A, Hisanaga T and Kinoshita M: Plasma brain natriuretic peptide as a biochemical marker of high left ventricular end-diastolic pressure in patients with symptomatic left ventricular dysfunction. Am Heart J 135: 825-832, 1998. 
27. Seronde MF, Gayat E, Logeart D, Lassus J, Laribi S, Boukef R, Sibellas F, Launay JM, Manivet P, Sadoune M, et al: Comparison of the diagnostic and prognostic values of B-type and atrial-type natriuretic peptides in acute heart failure. Int $\mathrm{J}$ cardiol 168 3404-3411, 2013.

28. Potocki M, Breidthardt $\mathrm{T}$, Reichlin $\mathrm{T}$, Hartwiger $\mathrm{S}$, Morgenthaler NG, Bergmann A, Noveanu M, Freidank H, Taegtmeyer AB, Wetzel K, et al: Comparison of midregional pro-atrial natriuretic peptide with N-terminal pro-B-type natriuretic peptide in the diagnosis of heart failure. J Intern Med 267: 119-129, 2010.

29. Lip GY and Gibbs CR: Does heart failure confer a hypercoagulable state? Virchow's triad revisited. J Am Coll Cardiol 33: 1424-1426, 1999.

30. Rengo G, Pagano G, Squizzato A, Moja L, Femminella GD, de Lucia C, Komici K, Parisi V, Savarese G, Ferrara N, et al: Oral anticoagulation therapy in heart failure patients in sinus rhythm: A systematic review and meta-analysis. PLoS One 8: e52952, 2013.

31. Camp NP, Jones SD, Liebeschuetz JW, et al: Serine protease inhibitors. Journal 2005

32. Askari AT, Brennan ML, Zhou X, Drinko J, Morehead A, Thomas JD, Topol EJ, Hazen SL and Penn MS: Myeloperoxidase and plasminogen activator inhibitor 1 play a central role in ventricular remodeling after myocardial infarction. J Exp Med 197: 615-624, 2003.
33. XuZ, Castellino FJ and Ploplis VA: Plasminogen activator inhibitor-1 (PAI-1) is cardioprotective in mice by maintaining microvascular integrity and cardiac architecture. Blood 115: 2038-2047, 2010.

34. Spiroski I, Kedev S, Antov S, Trajkov D, Petlichkovski A, Dzhekova-Stojkova S, Kostovska S and Spiroski M: Investigation of SERPINE1 genetic polymorphism in Macedonian patients with occlusive artery disease and deep vein thrombosis. Kardiol Pol 67: 1088-1094, 2009.

35. Iwanaga Y, Aoyama T, Kihara Y, Onozawa Y, Yoneda T and Sasayama S: Excessive activation of matrix metalloproteinases coincides with left ventricular remodeling during transition from hypertrophy to heart failure in hypertensive rats. J Am Coll Cardiol 39: 1384-1391, 2002

36. Janicki JS and Brower GL: The role of myocardial fibrillar collagen in ventricular remodeling and function. J Card Fail 8: (Suppl 6) S319-S325, 2002.

37. Zheng J, Chen Y, Pat B, Dell'italia LA, Tillson M, Dillon AR, Powell PC, Shi K, Shah N, Denney T, et al: Microarray identifies extensive downregulation of noncollagen extracellular matrix and profibrotic growth factor genes in chronic isolated mitral regurgitation in the dog. Circulation 119: 2086-2095, 2009.

38. Chatila K, Ren G, Xia Y, Huebener P, Bujak M and Frangogiannis NG: The role of the thrombospondins in healing myocardial infarcts. Cardiovasc Hematol Agents Med Chem 5: 21-27, 2007 